\title{
Perturbative Charm Production and the Prompt Atmospheric Neutrino Flux in light of RHIC and LHC
}

\author{
Atri Bhattacharya, ${ }^{a, *}$ Rikard Enberg, ${ }^{b}$ Mary Hall Reno, ${ }^{c}$ Ina Sarcevic ${ }^{d, e}$ and Anna \\ Stasto $^{f}$ \\ a Space sciences, Technologies and Astrophysics Research (STAR) Institute, Université de Liège, \\ Bât. B5a, 4000 Liège, Belgium \\ ${ }^{b}$ Department of Physics and Astronomy, Uppsala University, \\ Box 516, Uppsala, 75120 Sweden \\ ${ }^{c}$ Department of Physics and Astronomy, University of Iowa, \\ 30 North Dubuque Street, Iowa City, IA, 52242 U.S.A. \\ ${ }^{d}$ Department of Physics, University of Arizona, \\ 1118 E. Fourth Street, Tucson, AZ, 85721 U.S.A. \\ ${ }^{e}$ Department of Astronomy and Steward Observatory, University of Arizona, \\ 933 North Cherry Avenue, Tucson, AZ, 85721 U.S.A. \\ ${ }^{f}$ Department of Physics, Pennsylvania State University, \\ University Park, PA 16802, U.S.A \\ E-mail: A.Bhattacharya@uliege.be, rikard.enberg@physics.uu.se, \\ mary-hall-reno@uiowa.edu, ina@physics.arizona.edu, astasto@phys.psu.edu
}

Prompt neutrinos due to the decay of charmed mesons produced in the atmosphere from cosmicray and atmospheric nuclei interactions may be a significant source of background to ultra-high energy neutrino searches above $10 \mathrm{TeV}$. We re-evaluate this flux using updated charm production cross-sections based on QCD parameters, the charm quark mass, and the range for the factorization and renormalization scales that provide the best description of this data at fixed target experiments, at RHIC, and at LHC. We find that the prompt neutrino flux is reduced from previous results in the literature by a factor between two and eight, depending on the energy. We discuss the implications of our results for current IceCube data.

40th International Conference on High Energy physics - ICHEP2020

July 28 - August 6, 2020

Prague, Czech Republic (virtual meeting)

\footnotetext{
${ }^{*}$ Speaker
} 


\section{Introduction}

Cosmic ray protons incident at the earth with energies exceeding hundreds of $\mathrm{TeV}$ interact with atmospheric nuclei leading to the production of mesons. These thereafter decay and produce neutrinos that form the dominant neutrino flux between energies spanning tens of $\mathrm{GeV}$ to hundreds of $\mathrm{TeV}$. The lower energy end of this spectrum - up to a few tens of $\mathrm{TeV}$ - is dominated by neutrinos from the decays of light mesons — pions and kaons - produced in the atmosphere (e.g. $\pi^{ \pm} \rightarrow \mu^{ \pm} v_{\mu} \rightarrow e^{ \pm} v_{e} v_{\mu} v_{\mu}$ ). The resulting neutrino flux between energies of $10 \mathrm{GeV}-1 \mathrm{TeV}$ has been observed by several experiments (see [1] for a review) over the last two decades. At higher energies, as the production of heavier mesons such as the charmed mesons $\left(D^{ \pm, 0}\right)$ becomes increasingly favoured kinematically, the proportion of neutrinos from the decay of these mesons in the total atmospheric neutrino flux gradually grows. Charmed mesons have shorter lifetimes in comparison to the lighter pions and kaons; therefore they decay promptly, without losing energy between production and decay, leading to a harder spectral shape for the resulting neutrino flux, called the prompt neutrino flux. At energies of about $100 \mathrm{TeV}$, this flux starts dominating over the conventional neutrino population.

The understanding of prompt neutrino production assumes special relevance in light of recent successes of IceCube towards detecting ultra-high energy neutrinos extending from tens of $\mathrm{TeV}$ to a few PeV [2, 3], where it might be the key background beyond $100 \mathrm{TeV}$ energies. Unfortunately, this understanding is hampered by the large uncertainties in the QCD modeling of heavy meson production: $p N \rightarrow c \bar{c} X$. Within the realm of perturbative $\mathrm{QCD}$, uncertainties arise from from multiple facets: $a$ ) the charm mass $\left.\left(m_{c}\right), b\right)$ the factorization $\left(M_{F}\right)$ and renormalization $\left(\mu_{R}\right)$ scales, and $c$ ) the choice of parton distribution functions (PDFs). Hadronization of charm to produce $D$ mesons may be modeled by different phenomenological models, adding to the uncertainty. Finally, computing the prompt lepton flux involves folding the charmed hadron production cross-section with the nucleon flux in the incoming cosmic-ray flux, thereby incurring large uncertainties from the limited understanding of the extremely high-energy cosmic ray composition.

The previous benchmark for UHE prompt neutrino flux [4] used the dipole model for their computation. Our focus in this talk will be on recomputing this flux to incorporate improvements in the understanding of parameters describing perturbative charm production thanks to recent collider data and more reliable, state-of-the-art cosmic-ray models. We will discuss the implications of these revised estimates of the prompt flux for ultra-high energy experiments like IceCube.

\section{Charm production cross section}

Uncertainties in the parameters describing $p p \rightarrow c \bar{c} X$ are now better understood thanks to the measurement of the charm production cross-section at LHC [5-7] and RHIC [8, 9]. In addition, modern parton distribution functions are less uncertain at smaller $x$, in part thanks to collider data at higher and higher energies. A combination of these factors enables the determination of the charm production cross section more accurately than ever before.

Specifically, we use the range of factorization and renormalization scales determined in Ref. [10] to best describe current charm production data in colliders: $M_{F} / m_{c}=1.3-4.3$ and $\mu_{R} / m_{c}=1.7-1.5$ with $M_{F}=2.1 m_{c}$ and $\mu_{R}=1.6 m_{c}$ as the central values when using the CT10 


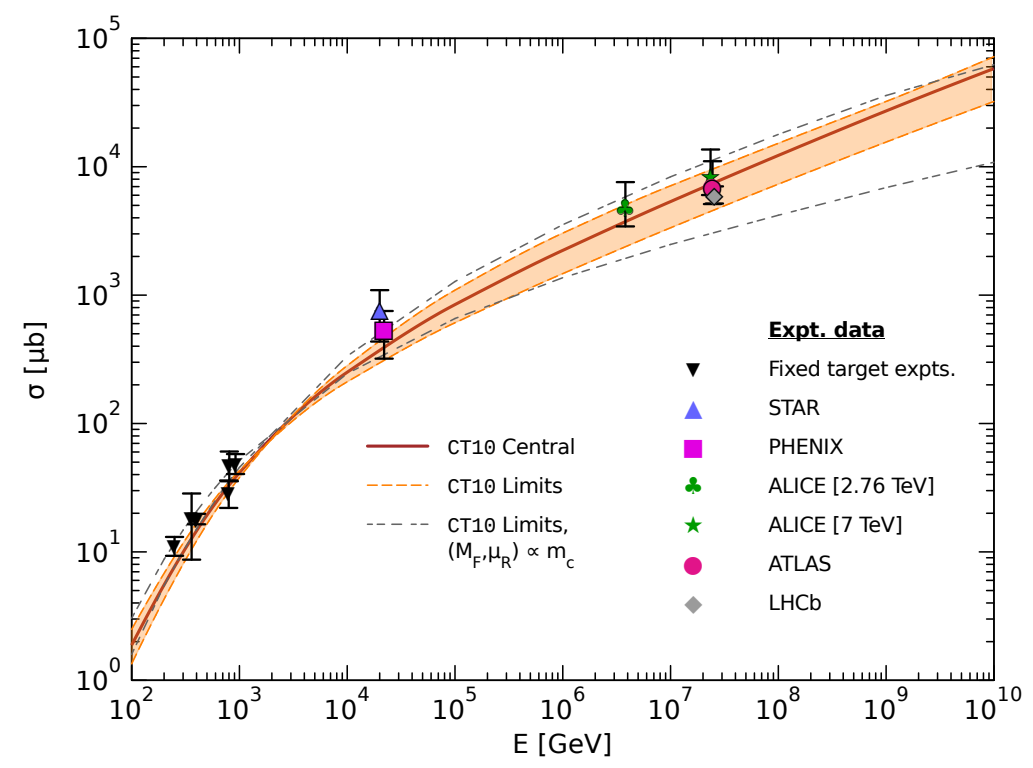

Figure 1: The charm production cross section $\sigma_{p N \rightarrow c \bar{c}+X}$ at NLO with $m_{c}=1.27 \mathrm{GeV}$ using the CT10 parton distributions for a range of scales described in the text. Also shown are the lower and upper limits (grey fine-dashed curves) when the scales are made to vary proportionally to $m_{c}$ rather than to $m_{T}$. For a list of experimental data shown here, see Ref. [12].

PDFs. We evolve the scales in multiples of the charm transverse mass $m_{T}^{2} \equiv\left(m_{c}^{2}+p_{T}^{2}\right)$, while using a fixed charm mass of $1.27 \mathrm{GeV}$, motivated by lattice QCD results. The charm production cross section is evaluated to the next-to-leading-order (NLO) in perturbative QCD [11]. Fig. 1 shows the resulting central curve for the charm production cross section as well as its range of uncertainty when varying the two scales withing the range described above.

\section{Prompt lepton flux}

The change in the flux of a particle $j$ as it traverses a slant depth $X$ in the atmosphere is described by the equation:

$$
\frac{\mathrm{d} \Phi_{j}}{\mathrm{~d} X}=-\frac{\Phi_{j}}{\lambda_{j}}-\frac{\Phi_{j}}{\lambda_{j}^{\mathrm{dec}}}+\sum_{k} S(k \rightarrow j)
$$

with flux losses described by the interaction (decay) length $\lambda\left(\lambda^{\mathrm{dec}}\right)$ and its regeneration from a different particle $k$ described by the generation function $S(k \rightarrow j)$, which is generically a function of both the particle energy $E_{k}$ and the slant depth $X$. To compute the lepton flux from the final neutrino flux we adopt a semi-analytical approach to solve these equations using spectrum-weighted $Z$-moments that depend on the energy alone. For example, the hadron production moments are defined by

$$
Z_{p h}\left(E_{h}\right)=\int_{x_{E_{\min }}}^{1} \frac{d x_{E}}{x_{E}} \frac{\phi_{p}^{0}\left(E_{h} / x_{E}\right)}{\phi_{p}^{0}\left(E_{h}\right)} \frac{1}{\sigma_{p A}\left(E_{h}\right)} \times A \frac{d \sigma}{d x_{E}}(p N \rightarrow h X),
$$


where, $A=14.5$ is the average atomic number of target air nuclei ${ }^{1}$,

$$
\frac{d \sigma}{d x_{E}}(p A \rightarrow h X)=A \int_{x_{E}}^{1} \frac{d z}{z} \frac{d \sigma}{d x_{c}}(p N \rightarrow c X) D_{c}^{h}(z)
$$

in terms of $x_{E}=E_{h} / E_{b}$ and $x_{c}=E_{c} / E_{b}=x_{E} / z$ for an incident cosmic ray nucleon energy (beam energy) $E_{b}$, and fragmentation functions $D_{c}^{h}$ assumed to be energy independent. The all-nucleon cosmic ray flux is described by $\phi_{p}(E, X) \simeq \phi_{p}^{0}(E) \exp \left(-X / \Lambda_{p}\right)$.

Previous results approximated the cosmic ray flux by a broken-power law [4]; here, we instead use a more recent parametrization from ref. [13] with fluxes from three populations: supernova remnants, other galactic sources, and extragalactic sources. The H3a flux has a mixed composition in the extragalactic population, while we designate the $H 3 p$ flux as the one where it is all protons (Fig. 2a).

We skip a full description of the procedure for going from the incident cosmic ray flux to the final prompt neutrino flux using $Z$-moments, and refer the reader to Ref. [12] instead. The resulting prompt neutrino fluxes for different choices of incident cosmic-ray flux and QCD parameters are shown in Fig. 2b.

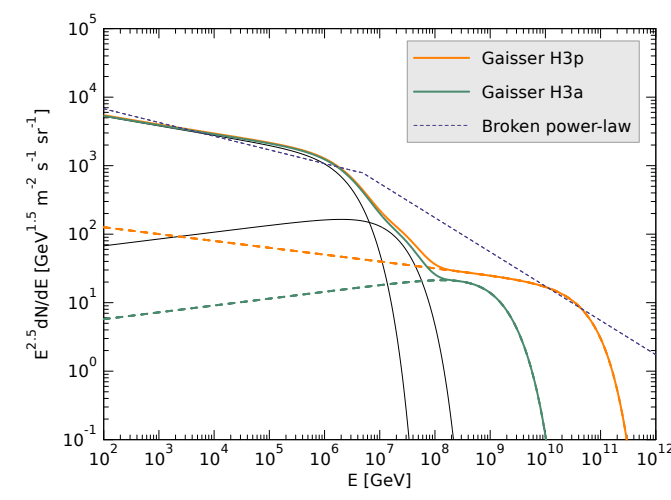

(a)

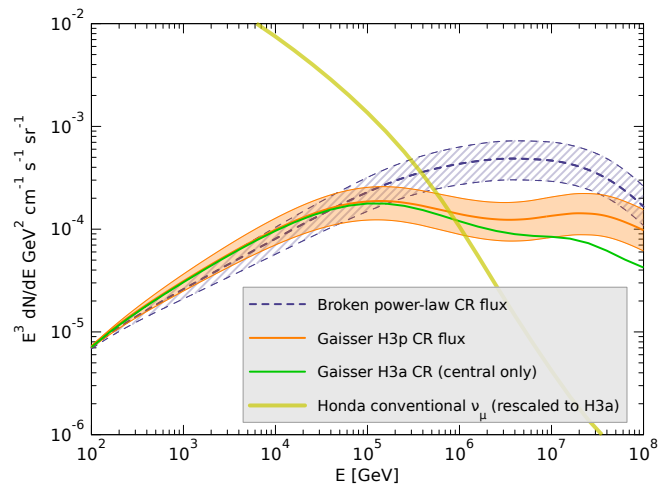

(b)

Figure 2: (a) The all-nucleon cosmic ray spectrum as a function of energy per nucleon for the three component model of ref. [13] with a mixed extragalactic population (H3a) and all proton extragalactic population (H3p), and for a broken power-law. (b) Our benchmark results for the prompt $v_{\mu}+\bar{v}_{\mu}$ flux scaled by $E^{3}$ (orange curve), using the $\mathrm{H} 3 \mathrm{p}$ cosmic ray flux. The blue curve uses instead a broken power-law (used in previous analyses, e.g., [4]).

\subsection{Comparison to previous results}

In comparison to the fluxes obtained in [4], our benchmark results, i.e. obtained using the Gaisser H3p cosmic ray flux and central values of the QCD parameters, are reduced by a factor ranging from 2 at lower energies (below $100 \mathrm{TeV}$ ) to a maximum of about 8 at high energies (at a few $\mathrm{PeV})$. Roughly quantifying the changes from the main ingredients in our calculation, we estimate that $a$ ) the use of updated cosmic-ray fluxes leads to a reduction in the prompt neutrino flux by

\footnotetext{
${ }^{1}$ We use the approximation $\sigma(p A \rightarrow c \bar{c} X) \simeq A \sigma(p N \rightarrow c \bar{c} X)$ throughout.
} 
about a factor $1.2-3$ at energies between $100 \mathrm{TeV}-1 \mathrm{PeV} ; b$ ) differences in the large $x$ behavior of the dipole and perturbative $\mathrm{QCD}$ evaluations of $p A \rightarrow c \bar{c} X$, account for the relative fluxes differing by a factor of $\sim 1.5$; and $c$ ) re-evaluation of $Z_{p p}$ moments and using updated $p$-Air cross section decreases the flux by about $30 \%$.

\section{Implications for IceCube}

Neutrinos produced in the atmosphere, whether conventionally via pion and kaon decay or via the prompt decay of heavier charmed mesons, are backgrounds to IceCube's searches for extragalactic neutrinos at ultra-high energies. Our calculation revises down existing prompt neutrino flux estimates significantly; consequently, this component of the background remains subdominant to the conventional flux up to energies of $100 \mathrm{TeV}$. Above these energies, the astrophysical flux overwhelms the atmospheric fluxes anyway; the prospect for prompt neutrino discovery at IceCube therefore remains low. We show the expected break-up of events based on their origins for the IceCube 988-day data [3] in Fig. 3.

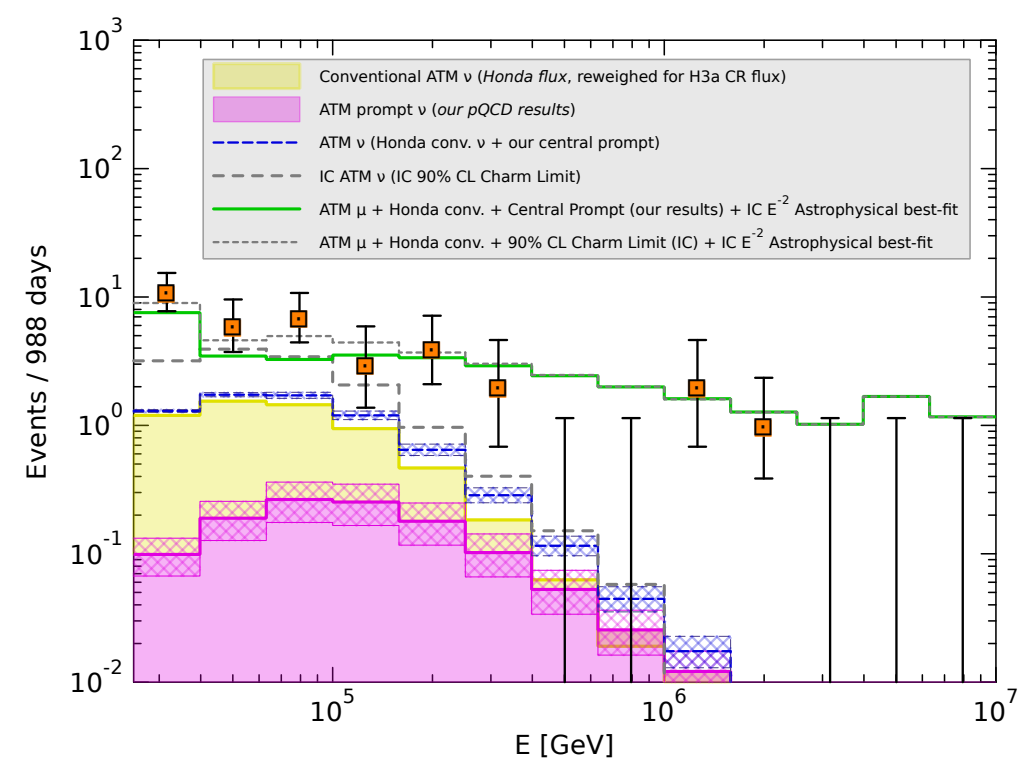

Figure 3: Event rates at IceCube from prompt neutrinos, with our updated prediction for the prompt flux indicated in magenta, along with uncertainties from variation in the QCD parameters indicated as a hatched region around the central curves. See Ref. [12] for details.

\section{References}

[1] T. K. Gaisser, Atmospheric neutrino fluxes, Phys. Scripta T 121 (2005) 51-56, [astro-ph/0502380].

[2] ICECuBE collaboration, M. Aartsen et al., Evidence for High-Energy Extraterrestrial Neutrinos at the IceCube Detector, Science 342 (2013) 1242856, [1311. 5238]. 
[3] ICECube collaboration, M. G. Aartsen et al., Observation of High-Energy Astrophysical Neutrinos in Three Years of IceCube Data, Phys.Rev.Lett. 113 (2014) 101101, [1405 . 5303].

[4] R. Enberg, M. H. Reno and I. Sarcevic, Prompt neutrino fluxes from atmospheric charm, Phys.Rev. D78 (2008) 043005, [0806. 0418].

[5] ALICE collaboration, B. Abelev et al., Measurement of charm production at central rapidity in proton-proton collisions at $\sqrt{s}=2.76 \mathrm{TeV}$, JHEP 1207 (2012) 191, [1205 . 4007].

[6] ATLAS collaboration, Measurement of $D^{(*)}$ meson production cross sections in $p p$ collisions at $\sqrt{s}=7 \mathrm{TeV}$ with the ATLAS detector, .

[7] LHCв collaboration, R. Aaij et al., Prompt charm production in pp collisions at sqrt $(s)=7$ TeV, Nucl.Phys. B871 (2013) 1-20, [1302 . 2864].

[8] PHENIX collaboration, A. Adare et al., Measurement of high-p $(T)$ single electrons from heavy-flavor decays in $p+p$ collisions at $\sqrt{s}=200-G e V$, Phys.Rev.Lett. 97 (2006) 252002, [hep-ex/0609010].

[9] STAR collaboration, L. Adamczyk et al., Measurements of $D^{0}$ and $D^{*}$ Production in $p+p$ Collisions at $\sqrt{s}=200 \mathrm{GeV}$, Phys.Rev. D86 (2012) 072013, [1204 . 4244].

[10] R. Nelson, R. Vogt and A. Frawley, Narrowing the uncertainty on the total charm cross section and its effect on the J/ $\psi$ cross section, Phys.Rev. C87 (2013) 014908, [1210 . 4610].

[11] M. Cacciari, S. Frixione and P. Nason, The $p(T)$ spectrum in heavy flavor photoproduction, JHEP 0103 (2001) 006, [hep-ph/0102134].

[12] A. Bhattacharya, R. Enberg, M. H. Reno, I. Sarcevic and A. Stasto, Perturbative charm production and the prompt atmospheric neutrino flux in light of RHIC and LHC, JHEP 06 (2015) 110, [1502.01076].

[13] T. K. Gaisser, Spectrum of cosmic-ray nucleons, kaon production, and the atmospheric muon charge ratio, Astropart.Phys. 35 (2012) 801-806. 